\title{
Increased concentrations of bone sialoprotein in joint fluid after knee injury
}

\author{
L Stefan Lohmander, Tore Saxne, Dick Heinegård
}

\begin{abstract}
Objective-To detect evidence for localised changes in bone matrix metabolism after joint trauma and in post-traumatic osteoarthritis by quantification of bone sialoprotein in joint fluid and serum after knee injury in a cross sectional study.

Methods-Samples of knee joint fluid and serum were obtained from volunteers with normal knees $(n=19)$, patients with rupture of the anterior cruciate ligament isolated or combined with tear of a meniscus $(n=114)$, and patients with isolated meniscus lesions $(n=80)$. Concentrations of bone sialoprotein were determined by ELISA. Concentrations of other markers of joint tissue metabolism in these samples were determined in previous investigations.
\end{abstract}

Results-The median concentration of bone sialoprotein in joint fluid from healthy volunteers was $122 \mathrm{ng} \mathrm{ml}^{-1}$ (range 41 to 183). Concentrations of bone sialoprotein were increased in both injury groups compared with the reference group (median for cruciate ligament injury $146 \mathrm{ng} \mathrm{ml}^{-1}$, range 72 to 339; median for meniscus injury $166 \mathrm{ng} \mathrm{m}^{-1}$, range 75 to 376). After injury, bone sialoprotein increased quickly and remained increased for six months. Bone sialoprotein in joint fluid was increased only in samples from joints with normal or nearly normal (fibrillated) cartilage, and was within reference range in joints with radiographic signs of osteoarthritis. Bone sialoprotein concentrations in joints with cruciate ligament injury were positively correlated with levels of aggrecan and cartilage oligomeric matrix protein fragments, and with levels of stromelysin-1 and tissue inhibitor of metalloproteinase-1. The ratios between the concentrations of bone sialoprotein in joint fluid and serum were $>1$ in the majority of the cruciate ligament injury cases.

Conclusions-The release of significant amounts of bone sialoprotein into joint fluid in connection with acute joint trauma may be associated with injury to, and active remodelling of, the cartilagebone interface and subchondral bone. The findings are consistent with dramatic shifts in cartilage, bone, and synovial metabolism following joint injury. Bone sialoprotein concentrations in synovial fluid may be a useful marker of subchondral injury and remodelling following joint injury.

(Ann Rheum Dis 1996;55:622-626)

Joint injury is associated with an increased rate of turnover of a number of macromolecules of articular cartilage and joint tissues. ${ }^{1}$ Thus the increased release into synovial fluid of fragments of matrix molecules, stromelysin (MMP-3), collagenase (MMP-1), and tissue inhibitor of metalloproteinases (TIMP-1) in both the acute and chronic phases after joint injury have been shown in cross sectional and longitudinal investigations. ${ }^{2-8}$ These changes in cartilage matrix metabolism after injury may be relevant to the increased risk for development of osteoarthritis in these patients. ${ }^{9}$

Assays of fragments of bone matrix molecules in joint fluid, serum, and urine suggest that changes occur also in bone matrix metabolism in osteoarthritis and rheumatoid arthritis. ${ }^{10-12}$ These findings are consistent with the suggestions that changes in metabolism, structure, and function of both bone and cartilage are cardinal features in the pathology of osteoarthritis and rheumatoid arthritis.

Magnetic resonance imaging of the acutely injured knee often reveals subchondral bone lesions in the presence of normal plain radiographs and normal arthroscopic findings. ${ }^{13-17}$ These previously unrecognised lesions may induce long term changes in the structure and biomechanical properties of the joint and could be important for the future development of post-traumatic osteoarthritis.

Bone sialoprotein is a bone specific molecule produced by osteoblasts with an $\mathrm{Mr}$ of $57 \mathrm{kDa}$, which constitutes $12 \%$ of the non-collagenous proteins of bone matrix. ${ }^{18-20}$ The function of bone sialoprotein is unknown, but it binds tightly to hydroxyapatite and to cells. The protein backbone of $33.6 \mathrm{kDa}$ contains an $\mathrm{RGD}$ sequence, which can mediate binding to an integrin, the $\alpha_{v} \beta_{3}$ receptor. ${ }^{21}$ The protein shows the highest level of synthesis at the cartilage-bone interface in the growth cartilage and is markedly enriched in the interface between articular cartilage and bone, the tidemark, and in the cement lines and mineralising osteoid. ${ }^{22-24}$ The primary cell expressing bone sialoprotein is an osteoblast, ${ }^{22}$ but it is also expressed by hypertrophic chondrocytes in the growth plate. ${ }^{25}$ 
Patient characteristics. Median (range) are given for age (ears), time (weeks after injury), volume (ml).

\begin{tabular}{llllll}
\hline Group & $n$ & Males & Age & Time & Vol \\
\hline Control & 19 & 14 & $\begin{array}{l}29 \\
(17-43)\end{array}$ & - & $2(1-5)$ \\
ACL & 114 & 80 & $\begin{array}{l}32 \\
(14-67)\end{array}$ & $\begin{array}{l}105 \\
(0-1250)\end{array}$ & $6(1-70)$ \\
Meniscus & 80 & 70 & $\begin{array}{l}45 \\
(16-70)\end{array}$ & $\begin{array}{l}44 \\
(0-1600)\end{array}$ & $5(1-90)$ \\
\hline
\end{tabular}

$\mathrm{ACL}$, anterior cruciate ligament injury group; Meniscus, meniscus injury group.

To identify alterations particularly at the bone-cartilage interface, we have assayed bone sialoprotein concentrations in joint fluid and serum after knee injury in a cross sectional study to detect evidence for localised changes in bone matrix metabolism after joint trauma and in post-traumatic osteoarthritis.

\section{Methods}

Samples of knee joint fluid and serum were obtained from 194 patients and 19 volunteers with healthy knees (control group). In this cross sectional study, each volunteer and patient supplied a sample at one time point only. For patients, sampling took place at varying times after knee injury or the beginning of symptoms. The control group has been described in more detail previously. ${ }^{26}$ The study groups were: anterior cruciate ligament rupture isolated or combined with a tear of meniscus or collateral ligament (ACL group), and isolated meniscus lesion (meniscus group) (table). Diagnosis was made by arthroscopy, radiography, and clinical examination. No patient was treated by surgery before serum or joint fluid sampling. Pharmacological treatment was limited to the occasional analgesic or non-steroidal anti-inflammatory drug. The degree of joint changes was assessed by arthroscopy and standing radiographs and scored on a scale of 1 to 10 , where 1 represents a normal joint by arthroscopy and radiography, 2-5 represents increasing degrees of joint cartilage changes by arthroscopy in a joint with a normal radiograph, and 6-10 increasing degrees of radiographic joint space loss on standing radiographs consistent with osteoarthritis. ${ }^{27}$ All procedures were approved by the ethics review board of the University.

Bone sialoprotein in joint fluid and serum was quantified by immunoassay, using a rabbit anti-human-bone sialoprotein antibody and human bone sialoprotein as standard. ${ }^{12}$ Intra-assay variation for both synovial fluid and serum was $<6 \%$, and interassay variation $<10 \%$. Bone sialoprotein reactivity in this immunoassay is not changed by repeated freeze-thawing of joint fluid or serum. Aggrecan fragments (AGN), cartilage oligomeric matrix protein (COMP), C-propeptide of collagen type II (PC2C), MMP-1, MMP-3, and TIMP-1 were previously assayed in these samples by dye precipitation or immunoassays, respectively. $^{2-5}$ Significance of differences between groups were determined by MannWhitney test or Kruskal-Wallis one way analysis of variance on ranks where appropriate, with comparison of subgroups $v$ the control group by Dunn's method. Correlations were estimated by Spearman rank correlation test. A $P$ value $<0.05$ was considered significant for differences and correlations, and all differences and correlations discussed as such are significant at this level or better.

\section{Results}

JOINT FLUID

Concentrations of bone sialoprotein were increased in both injury groups compared with control (fig 1). The median concentration of bone sialoprotein in joint fluid from the control group was $121.6 \mathrm{ng} \mathrm{ml}^{-1}$ (range 40.8 to 183.3). Reference values for bone sialoprotein in joint fluid have not previously been published.

After knee injury, bone sialoprotein concentrations quickly increased and were raised for up to two years after injury (fig 2). At later times, concentrations were not different from those in the control group. Concentrations did not differ between ACL and meniscus injuries in the various subgroups, and the temporal concentration patterns were similar in these two different types of joint injury.

When bone sialoprotein concentrations in samples obtained later than 26 weeks after injury (fig 2) were related to osteoarthritis stage, only those samples obtained from normal or nearly normal (fibrillated cartilage) joints (with regard to arthroscopy and radiography findings) had increased median concentrations (fig 3). At more advanced stages of cartilage changes, including those joints with radiographic signs of osteoarthritis, median concentrations of bone sialoprotein did not differ from the REF group.

Bone sialoprotein concentrations in joint fluid samples from patients with anterior cruciate ligament injury (ACL group) were positively correlated with AGN, COMP, MMP-3, and TIMP-1 (but not MMP-1 or PC2C) levels, with $r_{\mathrm{s}}$ values of $0.60,0.58,0.50$, and 0.54 , respectively, and with $\mathrm{P}$ values < 0.001 (fig 4, data shown only for COMP). In

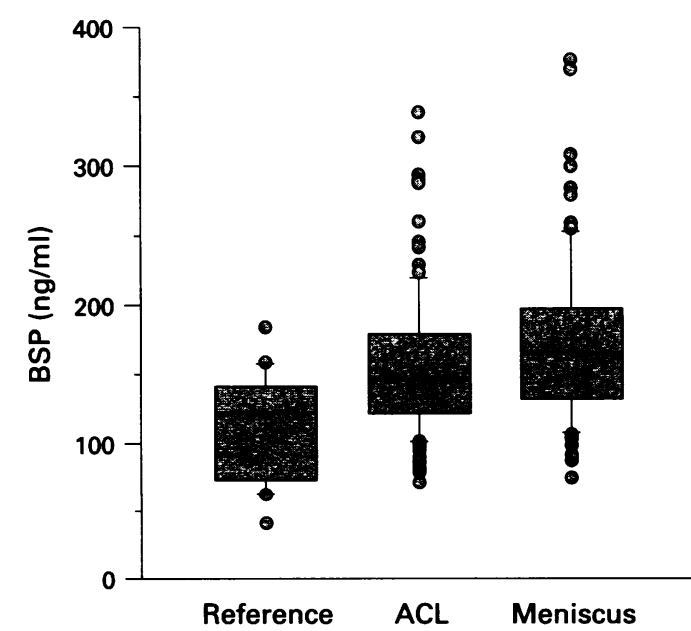

Figure 1 Bone sialoprotein (BSP) in joint fluid in the different study groups. Box represents 25th-75th centiles, line median, whiskers 10th and 90th centiles, symbols represent individual values outside those limits. Both injury groups differed from the control group $(P<0.001)$; the meniscus injury group differed from the cruciate ligament injury group $(P=0.027)$. 
contrast, bone sialoprotein concentrations in joint fluids from patients with meniscus tears did not correlate well enough with any of these markers to give $r_{s}$ values greater than $0.5\left(r_{s}\right.$ values between 0.25 and 0.08 , with $P$ values between 0.03 and 0.5). However, correlations between AGN and COMP or between MMP-3 and MMP-1 concentrations, were similarly good in these two diagnostic groups with $r_{s}$ values of 0.7 and 0.8 , respectively, and $P$ values < 0.001 .

\section{SERUM}

The median serum concentration of bone sialoprotein in the ACL group (age range 14 to 67 years) was $114 \mathrm{ng} \mathrm{ml}^{-1}$ (range 70-220 ng $\mathrm{ml}^{-1}$ ), compared with a median concentration of $75 \mathrm{ng} \mathrm{ml}^{-1}$ for a previously published healthy control group aged 21-70 years. ${ }^{12}$ Concentrations of bone sialoprotein in serum did not change in relation to the time after injury or

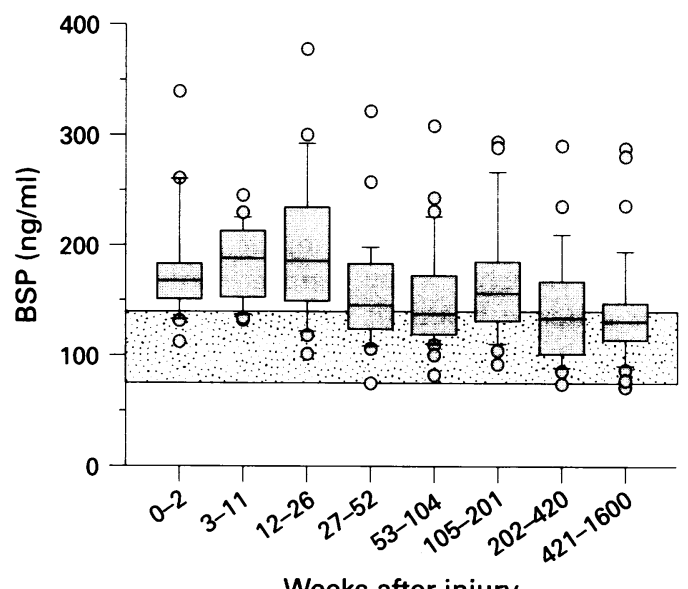

Figure 2 Bone sialoprotein (BSP) in joint fluid after cruciate ligament and/or meniscus lesion. Patients ordered by time after injury and divided into time windows indicated on horizontal axis. The numbers of patients in the subgroups were $23,19,20,25,29,22,22$, and 34 , respectively. The BSP concentrations in first five subgroups (up to 104 weeks after injury) were increased as compared to the control group $(P<0.001)$. Shaded zone indicates 25th-75th centiles for control group. See fig 1 for legend.

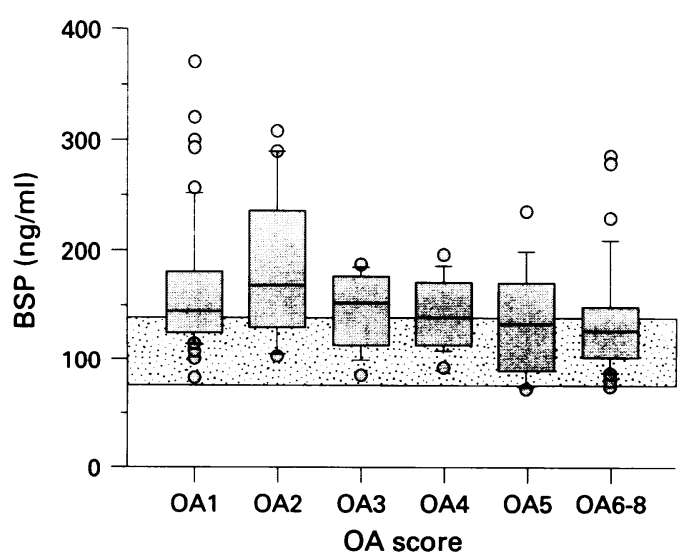

Figure 3 Bone sialoprotein (BSP) in joint fluid after cruciate ligament and/or meniscus lesion in relation to osteoarthritis (OA) stage by arthroscopy and radiography. Samples collected within 26 weeks of knee injury were excluded. The number of patients in the subgroups were 48 , $18,12,13,13$, and 29 , respectively. The $B S P$

concentrations in the first two subgroups (OA scores 1 and

2) were increased as compared to the control group $(P<$

$0.001)$. Shaded zone indicates 25th-75th centiles for

reference group. See fig 1 for legend. stage of osteoarthritis and did not correlate with serum concentrations of keratan sulphate or stromelysin-1 (data not shown).

The ratios between the concentrations of bone sialoprotein in joint fluid and serum were $>1$ in 45 out of the 53 cases (median ratio 1.3 for this group, 1.0 for the remaining eight patients) where joint fluid and serum from the same patient were assayed. The ratios between synovial fluid and serum concentrations of bone sialoprotein did not change in relation to time after injury (fig 5) or in relation to OA stage (data not shown). Interestingly, the range of ratios of bone sialoprotein in synovial fluid to bone sialoprotein in serum increased markedly with time after injury (Fig. 5).

\section{Discussion}

Changes in metabolism, structure, and function of bone and cartilage are prominent features in the pathology of both osteoarthritis and rheumatoid arthritis. In primary osteoarthritis as well as in post-traumatic oste-

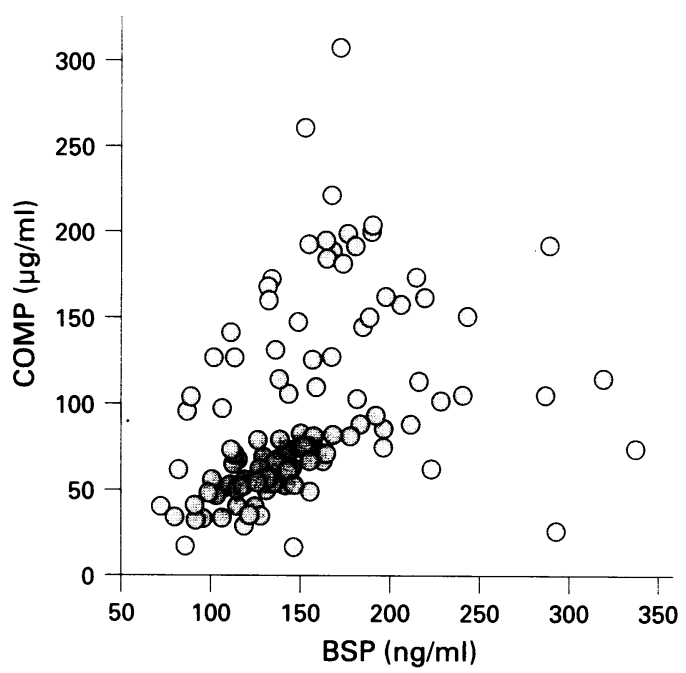

Figure 4 Correlation between concentrations of bone sialoprotein (BSP) and COMP in joint fluid samples from patients with anterior cruciate ligament $(A C L)$ injury $\left(r_{s}=\right.$ $0.58, P<0.001, n=108$ ).

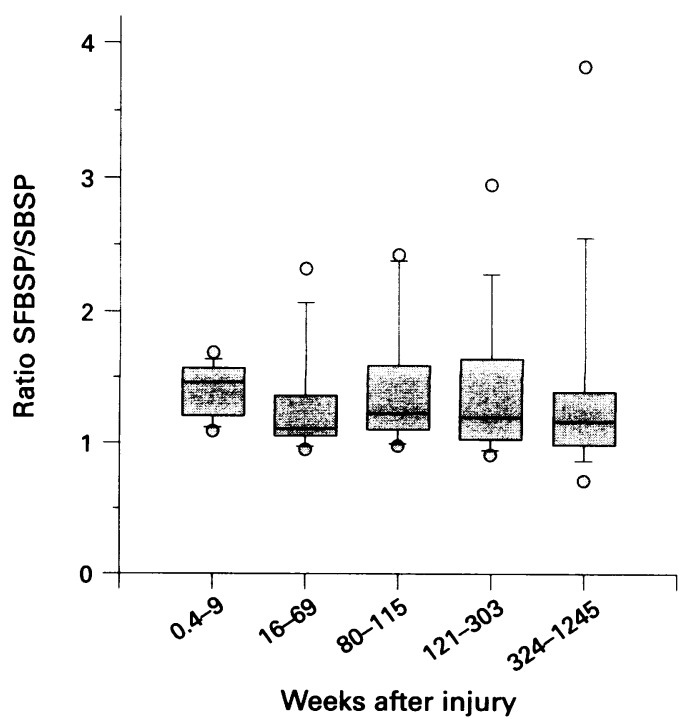

Figure 5 Ratios of concentrations of bone sialoprotein (BSP) in joint fluid and serum in relation to time after injury for patients with anterior cruciate ligament $(A C L)$ injury. See fig 1 for legend. The numbers of patients in the subgroups were 10,11,10,11, and 11, respectively. 
oarthritis, changes in subchondral bone structure and metabolism are early events in disease development, and active subchondral bone turnover in the osteoarthritic knee may signify an increased risk for disease progression. ${ }^{28}{ }^{29}$ The subchondral bone lesions in the injured knee frequently revealed by magnetic resonance imaging or bone scintigraphy, ${ }^{13-17}$ could also be associated with the future development of post-traumatic osteoarthritis.

The increased bone matrix turnover associated with osteoarthritis and joint injury usually results in an increased bone mineral density. In contrast, local resorption of subchondral bone and a generalised decrease in bone density are frequent findings in rheumatoid arthritis. ${ }^{30-32}$ In a previous study, synovial fluid concentrations of bone sialoprotein fragments in rheumatoid arthritis patients were shown to increase with increasing degrees of radiologically detected knee joint damage, while concentrations of another bone matrix protein, osteocalcin, were not related to the degree of joint damage. ${ }^{12}$ It was concluded that these increased levels of bone sialoprotein in rheumatoid arthritis synovial fluid most probably reflect an increased turnover rate of bone sialoprotein, which may in turn reflect the degree of disease involvement of a bone matrix compartment rich in bone sialoprotein.

In the present study, we detected significant increases in joint fluid concentrations of fragments of this bone specific protein in connection with knee injury. The release of significant amounts of this protein to joint fluid in connection with acute joint trauma and in the months following may be associated with injury to, and active remodelling of, the cartilage-bone interface and the subchondral bone in the period following the trauma. ${ }^{3233}$ Joint fluid bone sialoprotein concentrations in patients with cruciate ligament injury correlated well with concentrations of aggrecan and COMP fragments, and with MMP-3 and TIMP-1 concentrations. This is consistent with the notion that dramatic shifts in both cartilage, bone, and synovial metabolism occur following this injury, and that these changes may be causally related. Since the ratio of bone sialoprotein in synovial fluid to that in serum was greater than 1 , it is reasonable to conclude that a significant proportion of the bone sialoprotein in joint fluid is locally derived. There was a lesser degree of correlation between joint fluid concentrations of bone sialoprotein on the one hand, and aggrecan, COMP, MMP-3, and TIMP-1 concentrations on the other in samples from patients with meniscus injury. This is consistent with the different injury mechanisms in anterior cruciate ligament and meniscus injuries, and with previously shown differences in marker release for these two patient groups. ${ }^{34}$

Bone sialoprotein concentrations in joint fluid following injury and during development of post-traumatic osteoarthritis were raised only in the normal or near normal joint (with regard to arthroscopy and plain radiography findings). This is in agreement with the finding that median bone sialoprotein concentrations were increased only up to about two years after injury. Perhaps this corresponds to the period of most active remodelling of joint bone and tidemark after injury. The fact that the concentrations in joint fluid of fragments of cartilage matrix molecules such as AGN and COMP remain increased for much longer periods after injury is consistent with a more extensive metabolic perturbation of cartilage metabolism and its ultimate destruction in osteoarthritis. ${ }^{34}$

In contrast to rheumatoid arthritis, bone sialoprotein concentrations did not increase with increasing joint destruction during developing post-injury osteoarthritis. This difference may be due to the differences in juxta-articular bone remodelling and destruction in these two conditions. ${ }^{3031}$ It is possible that major alterations at the cartilage-bone interface occur early in those injuries which lead to progressive osteoarthritis. Notably, the levels of joint fluid bone sialoprotein showed a wide distribution early after injury. It may be that the cases with high bone sialoprotein levels will show a high future incidence of osteoarthritis.

We conclude that bone sialoprotein concentrations in synovial fluid may be a useful marker of subchondral injury and remodelling in association with joint trauma. The hypothesis that a subchondral injury may predispose to the development of posttraumatic osteoarthritis, and that bone sialoprotein release may be a marker of such injury, could be tested by, for example, prospective studies on knee injury patients monitored by ${ }^{99} \mathrm{Tc}-\mathrm{MDP}$ scintigraphy, radiography, magnetic resonance imaging, and bone sialoprotein concentrations in joint fluid.

Supported by the Swedish Medical Research Council, Lund University, the King Gustaf V 80-year Fund, the Craaford Foundation, Margaret and Axel Ax:son Johnson Foundation, Zoega Foundation, Kock Foundation, Nanna Svartz Zoega Foundation, Kock Foundation, Nanna Svartz Reumatism, Trygg Hansa, Astra Läkemedel, CIBA-GEIGY Reumatism, Trygg Hansa, Astra Läkem
and Merck Research Laboratories, USA.

1 Lohmander LS. Articular cartilage and osteoarthrosis-the role of molecular markers to monitor breakdown, repair role of molecular markers to monitor

2 Lohmander LS, Hoerrner LA, Lark MW. Metalloproteinases, tissue inhibitor and proteoglycan fragments in knee synovial fluid in human osteoarthritis. Arthritis Rheum 1993;36:181-9.

3 Lohmander LS, Hoerrner LA, Dahlberg L, Roos H, Björnsson S, Lark MW. Stromelysin, tissue inhibitor and proteoglycan fragments in human knee joint fluid after injury. $¥$ Rheumatol 1993;20:1362-8.

4 Lohmander LS, Saxne T, Heinegård D. Release of cartilage oligomeric matrix protein (COMP) into joint fluid after injury and in osteoarthrosis. Ann Rheum Dis 1994;53:8 injury.

5 Lohmander L S, Shinmei M. Procollagen II C-propeptide in joint fluid - changes in concentration with age and time after injury [abstr]. Trans Orthop Res Soc 1994;19:196.

6 Dahlberg L, Fridén T, Lark M W, Hoerrner L A Lohmander L S. Longitudinal study of cartilage matrix metabolism in patients with cruciate ligament rupture synovial fluid concentrations of aggrecan fragments, stromelysin-1 and tissue inhibitor of metalloproteinase-1. Br $\mathcal{f}$ Rheumatol 1994;33:1107-11.

7 Lohmander LS, Hoerrner LA, Lark MW. Increased concentrations of collagenase (MMP-1) in joint fluid after knee trations of collagenase (MMP-1) in joint fluid after knee injury and in

8 Lohmander LS, Ionescu M, Poole AR. Changes in aggrecan structure and metabolism after knee injury and in osteoarthritis [abstr]. Trans Orthop Res Soc 1995;20:412.

9 Lohmander LS, Roos H. Knee ligament injury, surgery and osteoarthrosis - truth or consequences? Acta Orthop Scand 1994;65:605-9. 
10 Campion GV, Delmas PD, Dieppe PA. Serum and synovia osteocalcin (bone Gla protein) levels in joint disease. $\mathrm{Br} f$ Rheumatol 1989;28.393-8.

11 Uebelhart D, Gineyts E, Chapuy M-C, Delmas PD. Urinary excretion of pyridinium crosslinks: a new marker of bone resorption in metabolic bone disease. Bone Miner 1990; 8:87-96.

12 Saxne T, Zunino L, Heinegård D. Increased release of bone sialoprotein into synovial fluid reflects tissue destruction in rheumatoid arthritis. Arthritis Rheum 1995;38:82-90.

13 Rosen MA, Jackson DW, Berger PE. Occult osseous lesions documented by magnetic resonance imaging associated with anterior cruciate ligament ruptures. Arthroscopy with anterior

14 Speer KP, Spritzer CE, Goldner JL, Garrett WE. Magnetic resonance imaging of traumatic knee articular cartilage injuries. Am ₹ Sports Med 1991;19:396-401.

15 Graf BK, Cook DA, De Smet AA, Keene JS. Bone Bruises on magnetic resonance imaging evaluation of anterior cruciate ligament injuries. Am $₹$ Sports Med 1993;21:220-3.

16 Spindler KP, Schils JP, Bergfeld JA, Andrish JT, Weiker GG, Anderson TE, et al. Prospective study of osseous, articular, and meniscal lesions in recent anterior cruciate ligament tears by magnetic resonance imaging and arthroscopy. $\mathrm{Am}$ tears by magnetic resonance

17 Adalberth T, Roos H, Laurén M, Åkeson P, Sloth M, Johnsson $\mathrm{K}$, et al. MRI, scintigraphy and arthroscopy in acute traumatic hemarthrosis of the knee. Am $\mathcal{F}$ Sports Med (in press).

18 Oldberg Å, Franzén A, Heinegård D. The primary structure of a cell-binding bone sialoprotein. $\mathcal{f}$ Biol Chem 1988 263:19430-2.

19 Heinegard D, Oldberg $\AA$. Structure and biology of cartilage and bone matrix non-collagenous macromolecules. FASEB F 1989;3:2042-51.

20 Fisher LW, McBride OW, Termine JD, Young MF. Human bone sialoprotein: deduced protein sequence and chromosomal localization. $₹$ Biol Chem 1990;265:2347-51.

21 Oldberg A, Franzén A, Heinegard D, Piersbacher M Ruoslahti E. Identification of a bone sialoprotein recepto in osteosarcoma cells. $\mathcal{F}$ Biol Chem 1988;263:19433-

22 Hultenby $K$, Reinholt $F$, Norgård $M$, Oldberg $\AA$, Wendel $M$, Heinegard D. Distribution and synthesis of bone sialoprotein in metaphyseal bone of young rats show a distinctly different pattern from that of osteopontin. Eur $\mathcal{F}$ Cell Biol 1994;63:230-9.
23 Riminucci M, Silvestrini G, Bonucci E, Fisher LW, Robey PG, Bianco $P$. The anatomy of bone sialoprotein immunoreactive sites in bone as revealed by combined ultrastructural histochemistry and immunohistochemistry. Calcif Tissue Int 1995;57:277-84.

24 de Bri E, Reinholt FP, Heinegård D, Mengarelli-Widholm $\mathrm{S}$, Svensson $\mathrm{O}$. Bone sialoprotein in guinea pig osteoarthritis. Acta Orthop Scand 1995;(suppl 266)66:78-9.

25 Shen Z, Heinegard D, Sommarin Y. Distribution and expression of cartilage oligomeric matrix protein and bone sialoprotein show marked changes during rat femoral head development. Matrix 1994;14:773-81.

26 Roos H, Dahlberg L, Hoerrner LA, Lark MW, Thona EJ-MA, Shinmei $\mathbf{M}$, et al. Markers of cartilage matrix metabolism in human joint fluid and serum-the effect of exercise. Osteoarthritis Cartilage 1995;3:7-14.

27 Dahlberg L, Ryd L, Heinegård D, Lohmander LS Proteoglycan fragments in joint fluid-influence of arthrosis and inflammation. Acta Orthop Scand 1992;63:417-23.

28 Radin EL. Osteoarthrosis-the orthopedic surgeons perspective. Acta Orthop Scand 1995;66(suppl 266):6-9.

29 Dieppe P, Cushnagan J, Young P, Kirwan J. Prediction of the progression of joint space narrowing in osteoarthritis of the knee by bone scintigraphy. Ann Rheum Dis 1993; 52:557-63.

30 Hart DJ, Mootoosamy I, Doyle DV, Spector TD. The relationship between osteoarthritis and osteoporosis in the general population: the Chingford study. Ann Rheum Dis general population:

31 Cooper C, Poll V, McLaren M, O'n Daunt S, Cawley MID. Alterations in appendicular skeletal mass in patients with rheumatoid, psoriatic, and osteoarthropathy. Ann Rheum Dis 1988;47:481-4.

32 Donohue JM, Buss D, Oegema TR, Thompson RC. The effects of indirect blunt trauma on adult canine articular cartilage. F Bone foint Surg Am 1983;65A:948-57.

33 Vener MJ, Thompson RC, Lewis $\mathrm{L}$, Oegema TR Subchondral damage after acute transarticular loading: an in vitro model of joint injury. $f$ Orthop Res 1992;10:75965.

34 Lohmander LS, Roos H, Dahlberg L, Hoerrner LA, Lark MW. Temporal patterns of stromelysin, tissue inhibitor and proteoglycan fragments in synovial fluid after injury to knee cruciate ligament or meniscus. $f$ Orthop Res 1994;12:21-8. 